\title{
Target detection and classification in SAR images using region covariance and co-difference
}

\author{
Kaan Duman ${ }^{\mathrm{a}}$, Abdulkadir Eryildirim ${ }^{\mathrm{b}}$, A. Enis Cetin ${ }^{\mathrm{a}}$ \\ ${ }^{a}$ Dept. of Electrical and Electronics Engineering, Bilkent University, 06800, Ankara, Turkey \\ ${ }^{b}$ Meteksan Savunma Sanayii A.S., Ankara, Turkey
}

\begin{abstract}
In this paper, a novel descriptive feature parameter extraction method from synthetic aperture radar (SAR) images is proposed. The new approach is based on region covariance (RC) method which involves the computation of a covariance matrix whose entries are used in target detection and classification. In addition the region co-difference matrix is also introduced. Experimental results of object detection in MSTAR (moving and stationary target recognition) database are presented. The RC and region co-difference method delivers high detection accuracy and low false alarm rates. It is also experimentally observed that these methods produce better results than the commonly used principal component analysis (PCA) method when they are used with different distance metrics introduced.
\end{abstract}

Keywords: Synthetic aperture radar (SAR) images, automatic target recognition (ATR) and classification, region covariance $(\mathrm{RC})$, region co-difference matrix, principal component analysis (PCA)

\section{INTRODUCTION}

Automatic recognition and classification of man-made objects in SAR images have been an active research area because SAR sensors can produce images of the scenes in all weather conditions at any time of the day which is not possible with infrared and optical sensors ${ }^{[1,2]}$. There are many areas of application where the recognition of a target or texture signal is important including military combat identification, meteorological observation, battlefield surveillance, mining, oceanography, classification of earth terrain etc.

A typical and complete automatic target recognition (ATR) system includes five stages: detection, discrimination, classification, recognition, and identification (Figure 1). In some systems, only some of the above stages are available. In general, a typical SAR ATR system fulfils three functions: detection, recognition and identification.

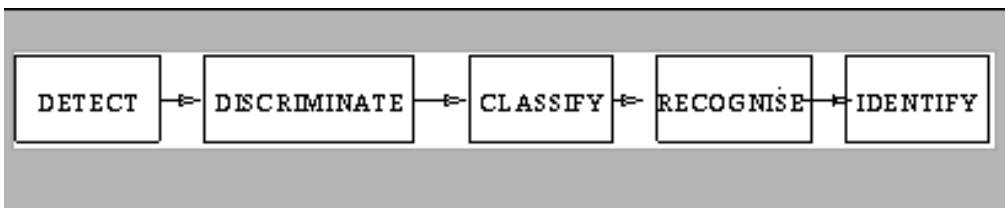

Fig 1. Stages of an ATR system

The use of the region covariance approach in a SAR ATR system is investigated in this paper. This method can be used in detection or discrimination stage of a SAR ATR system. The SAR images are first processed using a simple detector based on the amplitude information to determine regions of interest (ROIs). In ROIs, region covariance method is used to extract feature parameters.

The region covariance method is used to describe an image region with a covariance matrix in order to extract features for detection and classification problems. In recent years, region covariance is successfully used in face, human and various objects detection and classification problems ${ }^{[3,4]}$. In this work, this method is applied to SAR images to detect man-made objects.

Algorithms for Synthetic Aperture Radar Imagery XVI, edited by Edmund G. Zelnio, Frederick D. Garber, Proc. of SPIE Vol. 7337, 73370P · (c) 2009 SPIE · CCC code: 0277-786X/09/\$18 · doi: 10.1117/12.818725 
To detect an object, covariance matrices are compared to each other using a distance metric providing robust detection and classification. There are several advantages of using covariance features. First is that these features have small dimension and they are invariant to rotation, scale and illumination as these characteristics are all absorbed within the covariance matrix. In addition, the computation of covariance provides an average filter to filter out the speckle noise corrupting SAR images. In this work, a new region co-difference matrix is also introduced for object detection and classification which replace the covariance matrix in the novel RC method.

\section{REGION COVARIANCE (RC) MATRIX}

In this paper, to detect a man-made object on SAR images the area of interest is held as one region with one covariance feature. As in ${ }^{[4]}$, it is assumed that a single covariance feature is enough to discriminate in between distributions. For each pixel in the ROI, a seven-dimensional feature vector $z$ is computed as,

$$
z_{k}=\left[\begin{array}{lll}
x & y & I
\end{array}\left|\frac{d I(x, y)}{d x}\right|\left|\frac{d I(x, y)}{d y}\right|\left|\frac{d^{2} I(x, y)}{d x^{2}}\right|\left|\frac{d^{2} I(x, y)}{d y^{2}}\right|\right]^{T}
$$

where, $k$ is the label of a pixel, $(x, y)$ is the position of a pixel, $I$ is intensity of a pixel (as gray scale images are used in this work), $d(I(x, y) / d x$ is the horizontal and $d(I(x, y)) / d y$ is the vertical first derivatives of the ROI calculated through the filter [-1 01$]$ ],$d^{2}(I(x, y)) / d x^{2}$ is the horizontal and $d^{2}(I(x, y)) / d y^{2}$ is the vertical second derivatives of the ROI calculated through the filter $[-12-1]$. For a 4 by 4 region, $\mathrm{k}=1,2, \ldots 16 ; \mathrm{x}=-2,1,0,1 ; \mathrm{y}=-2,1,0,1$.

Let the feature vector $z$ be defined as:

$$
z_{k}=\left[z_{k}(i)\right]^{T}
$$

where, $i$ is the index of the feature vector. After gaining this feature vector, a fast method for computing the covariance matrices by using integral images is applied. This method features same calculation complexity for all window sizes after computing the integral images as provided in ${ }^{[5]}$. The 7 by 7 covariance matrix $C_{R}$ of a ROI $R$ is defined by the fast covariance matrix computation formula:

$$
C_{R}=\left[c_{R}(i, j)\right]=\left(\frac{1}{n-1}\left[\sum_{k=1}^{n} z_{k}(i) z_{k}(j)-\frac{1}{n} \sum_{k=1}^{n} z_{k}(i) \sum_{k=1}^{n} z_{k}(j)\right]\right)
$$

where, $n$ is the total number of pixels in the ROI and $c_{R}(i, j)$ is the $(i, j)^{\text {th }}$ component of the covariance matrix.

In order to match two regions these calculated covariance matrices are compared to each other using a distance metric based on generalized eigenvalues. Two images (or two ROIs $R_{l}$ and $R_{2}$ ) have a distance $\rho$ calculated with their covariance matrices using the following formula ${ }^{[3]}$ :

$$
\rho\left(C_{1}, C_{2}\right)=\sqrt{\sum_{i=1}^{p} \log ^{2} \lambda_{i}\left(C_{1}, C_{2}\right)}
$$

where $C_{1}, C_{2}$ are the covariance matrices of regions $R_{1}$ and $R_{2}$, respectively and $\lambda_{i}$ is the $i^{\text {th }}$ generalized eigenvalue and $p$ is the number of eigenvalues found which is 7 .

SAR image classification and detection is carried out using the distance metric defined in Equation (4). The covariance matrix of given ROI is compared to the covariance matrix of the target and clutter images in the training set. The image producing the smallest distance value determines the outcome. 


\section{REGION CO-DIFFERENCE MATRIX}

Computational cost of a single covariance matrix for a given image region is not heavy. However, computational cost becomes important when scanning a big region at different scales and all locations is wanted. Furthermore, many video processing applications require real-time solutions. In order to decrease the computational cost, the RC matrix definition in Equation (3) is modified to obtain the co-difference equation as in Equation (5).

$$
C_{R}=\left[c_{R}(i, j)\right]=\left(\frac{1}{n-1}\left[\sum_{k=1}^{n} z_{k}(i) \oplus z_{k}(j)-\frac{1}{n} \sum_{k=1}^{n} z_{k}(i) \oplus \sum_{k=1}^{n} z_{k}(j)\right]\right)
$$

where the scalar multiplication is replaced by an additive operator $\oplus$. The operator $\oplus$ is basically a summation operation but the sign of the result behaves similar to the multiplication operation.

$$
a \oplus b=\left\{\begin{array}{c}
\mathrm{a}+\mathrm{b}, \quad \text { if } a \geq 0 \text { and } b \geq 0 \\
\mathrm{a}-\mathrm{b}, \quad \text { if } a \leq 0 \text { and } b \geq 0 \\
-\mathrm{a}+\mathrm{b}, \quad \text { if } a \geq 0 \text { and } b \leq 0 \\
-\mathrm{a}-\mathrm{b}, \quad \text { if } a \leq 0 \text { and } b \leq 0
\end{array}\right\}
$$

for real numbers $a$ and $b$. Above equation can be expressed also as follows:

$$
a \oplus b=\operatorname{sign}(a \times b)(|a|+|b|)
$$

Since $a \oplus b=b \oplus a$, the co-difference matrix is also symmetric as the RC matrix. Co-difference matrix behaves similar to the region covariance function. If two variables tend to vary together, co-difference function produces positive results as the covariance. When two variables tend to vary inversely, co-difference equation gives negative results. On the other hand, replacing the multiplication operation with summation operation decreases computational cost.

The operator $\oplus$ satisfies totality, associativity and identity properties; therefore it is a monoid function. In other words it is a semigroup with identity property. Similar statistical methods are used in ${ }^{[8]}$. Another similar statistical function is the average magnitude difference function (AMDF), which is widely used in speech processing to determine the periodicity of voiced sounds.

\section{EXPERIMENTAL STUDIES}

\subsection{Database}

MSTAR SAR image database ${ }^{[6]}$ is used in this work, which consists of three military target and clutter images. These targets, which refer to man-made (metal) objects in this work, are BMP-2 and BTR-70 armored personal carriers, and T72 main battle tank. Examples of these images are shown in Figure 2. Each target image has a size of 128 by 128. Several of the clutter images of size 128 by 128 are also shown in Figure 2. They are cropped from MSTAR images whose actual sizes are 1476 by 1784 . 


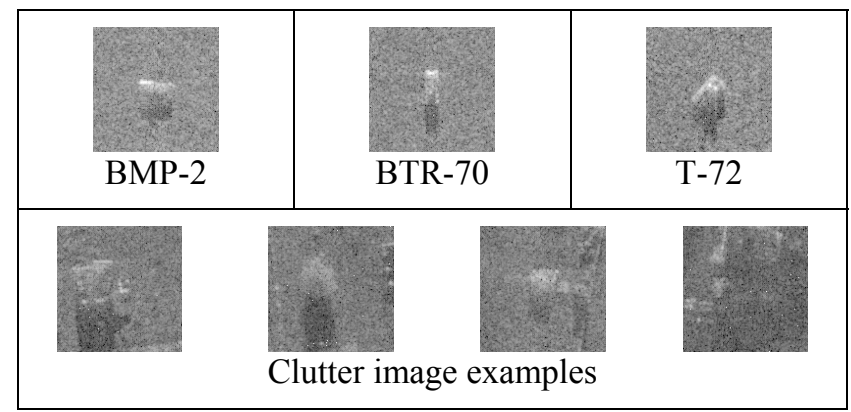

Fig. 2 Some MSTAR target images and clutter examples

Using MSTAR database, training and test sets are constructed with two classes containing targets and clutter respectively. Table 1 shows the number of samples corresponding to each category:

Table 1. Number of images used in training and testing studies

\begin{tabular}{|c|c|c|}
\hline & Number of Training Process & Number of Testing Process \\
\hline Target & 108 & 2651 \\
\hline Clutter & 569 & 13445 \\
\hline
\end{tabular}

As inputs to the various methods presented below, these 128 by 128 images are used along with 64 by 64 image chips cropped from these images capturing the targets as demonstrated in Figure 3. These chips provide reduction in the size of the data.

\begin{tabular}{|c|c|c|c|}
\hline & \multicolumn{3}{|c|}{ Targets } \\
\hline Image Size & BMP-2 & BTR-70 & T-72 \\
\hline 128 by 128 & & & \\
\hline $\begin{array}{c}\text { 64 by } 64 \\
\text { (Zoomed) }\end{array}$ & Clutters & \\
\hline 128 by 128 & & & \\
\hline
\end{tabular}

Fig. 3 Examples of 128 by 128 and cropped 64 by 64 target and clutter images 


\subsection{Experimental Results}

The performance of the detection is determined according to two parameters: detection accuracy $=$ (number of correctly detected target images) / (number of total target images) and false alarm $=$ (number of clutter images detected as target images) / (number of total clutter images). Results delivering these values of the whole and target focused 64 by 64 images for the methods described are summarized in Tables 2, 3, 4, 5 and 6 in exact ratio and percentages form. Finally the target detection and classification simulation results for the former PCA method are presented in Table 7.

The following table shows the results in detection and classification of targets in SAR images using the region covariance (RC) matrix with the distance metric defined in (4).

Table 2. Detection accuracy and false alarm rates achieved using region covariance (RC) method

\begin{tabular}{|c|c|c|}
\hline Input images & & Rates \\
\hline \multirow{2}{*}{$\mathbf{1 2 8}$ by $\mathbf{1 2 8}$} & Detection accuracy of target images & $2643 / 2651(99.70 \%)$ \\
\cline { 2 - 3 } & False alarm rates (incorrectly classified clutter images) & $11 / 13445(0.082 \%)$ \\
\hline \multirow{2}{*}{$\mathbf{6 4}$ by $\mathbf{6 4}$} & Detection accuracy of target images & $2648 / 2651(99.89 \%)$ \\
\cline { 2 - 3 } & False alarm rates (incorrectly classified clutter images) & $3 / 13445(0.023 \%)$ \\
\hline
\end{tabular}

The database is also used to acquire the performance rates for detection and classification of targets in SAR images using region co-difference matrix and the distance metric defined in (4), i.e. the original distance metric. Following table presents the results.

Table 3. Detection accuracy and false alarm rates achieved using region co-difference matrix method

\begin{tabular}{|c|c|c|}
\hline Input images & & Rates \\
\hline \multirow{2}{*}{$\mathbf{1 2 8}$ by $\mathbf{1 2 8}$} & Detection accuracy of target images & $2648 / 2651(99.89 \%)$ \\
\cline { 2 - 3 } & False alarm rates (incorrectly classified clutter images) & $0 / 13445(0 \%)$ \\
\hline \multirow{2}{*}{$\mathbf{6 4}$ by 64 } & Detection accuracy of target images & $2648 / 2651(99.89 \%)$ \\
\cline { 2 - 3 } & False alarm rates (incorrectly classified clutter images) & $17 / 13445(0.126 \%)$ \\
\hline
\end{tabular}

The studies with the database are expanded such that new distance metrics are defined for comparison of the region covariance and co-difference matrices in order to improve the performance. The first modified distance metric is defined as the square root of the sum of the logarithmic square of the differences between respective eigenvalues of $C_{l}$ and $C_{2}$ as follows:

$$
\rho_{2}\left(C_{1}, C_{2}\right)=\sqrt{\sum_{i=1}^{p} \log ^{2}\left(\lambda_{i}\left(C_{1}\right)-\lambda_{i}\left(C_{2}\right)\right)}
$$

where, $C_{l}, C_{2}$ are the covariance matrices of regions $R_{1}$ and $R_{2}$, respectively and $\lambda_{i}$ is the $i^{\text {th }}$ eigenvalue of $C_{l}, C_{2}$ separately and $p$ is the number of eigenvalues for $C_{l}$ and $C_{2}$. The resulting performance rates using this distance metric is as follows: 
Table 4. Detection accuracy and false alarm rates achieved using RC and region co-difference matrix method with the distance metric defined in (8)

\begin{tabular}{|c|c|c|}
\hline Input images & Using region covariance matrix & Rates \\
\hline & Detection accuracy of target images & $2581 / 2651(97.36 \%)$ \\
\hline \multirow{2}{*}{$\mathbf{1 2 8}$ by $\mathbf{1 2 8}$} & False alarm rates (incorrectly classified clutter images) & $7 / 13445(0.052 \%)$ \\
\cline { 2 - 3 } & Detection accuracy of target images & $2592 / 2651(97.77 \%)$ \\
\hline \multirow{2}{*}{$\mathbf{6 4}$ by $\mathbf{6 4}$} & False alarm rates (incorrectly classified clutter images) & $25 / 13445(0.186 \%)$ \\
\hline & Using region co-difference matrix & \\
\hline \multirow{2}{*}{$\mathbf{1 2 8}$ by $\mathbf{1 2 8}$} & Detection accuracy of target images & $2509 / 2651(94.64 \%)$ \\
\cline { 2 - 3 } & False alarm rates (incorrectly classified clutter images) & $24 / 13445(0.179 \%)$ \\
\hline \multirow{2}{*}{$\mathbf{6 4}$ by $\mathbf{6 4}$} & Detection accuracy of target images & $2598 / 2651(98 \%)$ \\
\cline { 2 - 3 } & False alarm rates (incorrectly classified clutter images) & $33 / 13445(0.245 \%)$ \\
\hline
\end{tabular}

A new distance metric is also defined which is based on the distance between the trains' and tests' region covariance matrix values rather than the eigenvalues. This distance metric is defined by the following equation:

$$
\rho_{3}\left(C_{1}, C_{2}\right)=\sqrt{\sum_{i=1}^{p}\left[\sum_{j=1}^{p}\left(\frac{\left|C_{1}(i, j)-C_{2}(i, j)\right|}{C_{2}(i, i)}\right)\right]}
$$

where, $C_{1}, C_{2}$ are the covariance matrices of regions $R_{I}$ and $R_{2}$, respectively. For this case, $R_{I}$ is the region acquired from the test images, whereas $R_{2}$ is the region acquired from the train images. Using no eigenvalues and only looking to the sum of difference in between the test and train covariance matrix values significantly reduces the computational cost. Since the pixel intensity values and their derivatives have different scales of values, normalization is required to compare the covariance values of the two regions. The target detection and classification results of SAR images using this distance metric with region covariance matrices are shown in the following table:

Table 5. Detection accuracy and false alarm rates achieved using RC and region co-difference matrix method with the distance metric defined in (9)

\begin{tabular}{|c|c|c|}
\hline Input images & Using region covariance matrix & Rates \\
\hline & Detection accuracy of target images & $2647 / 2651(99.85 \%)$ \\
\hline \multirow{2}{*}{$\mathbf{1 2 8}$ by $\mathbf{1 2 8}$} & Detection accuracy of target images & $0 / 13445(0 \%)$ \\
\cline { 2 - 3 } & False alarm rates (incorrectly classified clutter images) & $2651 / 2651(100 \%)$ \\
\hline \multirow{2}{*}{$\mathbf{6 4}$ by $\mathbf{6 4}$} & False alarm rates (incorrectly classified clutter images) & $2 / 13445(0.015 \%)$ \\
\hline & Dsing region co-difference matrix & \\
\hline \multirow{2}{*}{$\mathbf{1 2 8}$ by $\mathbf{1 2 8}$} & Fetection accuracy of target images & $2649 / 2651(99.92 \%)$ \\
\cline { 2 - 3 } & False alarm rates (incorrectly classified clutter images) & $0 / 13445(0 \%)$ \\
\hline \multirow{2}{*}{$\mathbf{6 4}$ by $\mathbf{6 4}$} & Detection accuracy of target images & $2650 / 2651(99.96 \%)$ \\
\cline { 2 - 3 } & False alarm rates (incorrectly classified clutter images) & $0 / 13445(0 \%)$ \\
\hline
\end{tabular}

The new distance metric finds distance between two ROIs according to the normalization by only the covariance matrix of the training regions. This metric is also modified such that the normalization is made by contribution of both train and test images' covariance matrices. This metric is defined as in the following equation: 


$$
\rho_{3}\left(C_{1}, C_{2}\right)=\sqrt{\sum_{i=1}^{p}\left[\sum_{j=1}^{p}\left(\frac{\left|C_{1}(i, j)-C_{2}(i, j)\right|}{\left(C_{1}(i, i)+C_{2}(i, i)\right)}\right)\right]}
$$

where, $C_{l}, C_{2}$ are the covariance matrices of regions $R_{l}$ (from test images) and $R_{2}$ (from train images), respectively. The normalization is done again by the diagonal values of the covariance matrices. The target detection and classification results using this metric is as follows:

Table 6. Detection accuracy and false alarm rates achieved using RC method and co-difference matrix method with the distance metric defined in (10)

\begin{tabular}{|c|c|c|}
\hline Input images & Rates \\
\hline & Using region covariance matrix & \\
\hline \multirow{2}{*}{128 by $\mathbf{1 2 8}$} & Detection accuracy of target images & $2642 / 2651(99.66 \%)$ \\
\cline { 2 - 3 } & False alarm rates (incorrectly classified clutter images) & $0 / 13445(0 \%)$ \\
\hline \multirow{2}{*}{$\mathbf{6 4}$ by $\mathbf{6 4}$} & Detection accuracy of target images & $2651 / 2651(100 \%)$ \\
\cline { 2 - 3 } & False alarm rates (incorrectly classified clutter images) & $1 / 13445(\sim 0 \%)$ \\
\hline & Using region co-difference matrix & \\
\hline \multirow{2}{*}{$\mathbf{1 2 8}$ by 128 } & Detection accuracy of target images & $2651 / 2651(100 \%)$ \\
\cline { 2 - 3 } & False alarm rates (incorrectly classified clutter images) & $0 / 13445(0 \%)$ \\
\hline \multirow{2}{*}{$\mathbf{6 4}$ by $\mathbf{6 4}$} & Detection accuracy of target images & $2651 / 2651(100 \%)$ \\
\cline { 2 - 3 } & False alarm rates (incorrectly classified clutter images) & $0 / 13445(0 \%)$ \\
\hline
\end{tabular}

Finally, the commonly used principal component analysis (PCA) method is also applied to the MSTAR database. PCA is basically an eigenvector-based multivariate analysis method which involves extracting a smaller number of uncorrelated variables named principal components from a number of possibly correlated variables ${ }^{[9]}$. This method is widely applied in many types of analysis such as neuroscience, face recognition, image compression due to its simple, non-parametric nature and ability to reduce a data of high dimension to a lower dimension.

The implementation of PCA includes the following phases: First a dataset as an M by N matrix is formed from the training images of the database, where $\mathrm{M}$ is the number of samples and $\mathrm{N}$ is the vector length, i.e. data dimension of each sample. The mean is subtracted from each data dimension. The covariance of this new M by $\mathrm{N}$ matrix is calculated. Next the eigenvectors and eigenvalues of the covariance matrix are computed. Finally the determined number of principal components is selected to form a feature vector. The number of the principal components, which also corresponds to the length of the feature vector, is important and can be adjusted based on the dataset and performance results. Increasing the number of principal components may degrade the performance. In this work, using more than 8 principal components slightly reduce the target detection and classification performance. The best performance is achieved with 8 principal components, as listed in Table 7.

The support vector machine (SVM) is used in order to classify in between the feature vectors extracted from the training and test images of the database to detect and classify targets in SAR images. SVM is a supervised machine learning method based on the statistical learning theory and approach developed by Vladimir Vapnik. SVMs have been applied to SAR target classification problems and they have given superior performance measures than the other classifiers ${ }^{[10]}$. There exist many parameters corresponding to different SVM kernels. In this study, polynomial SVM kernel acquired from ${ }^{[1]}$ which provided the highest performance is used with default parameters (Table 7).

The best target detection and classification performance results of PCA method are presented in Table 7. These results are important to make comparisons in between our new method and the traditional PCA method. 
Table 7. Best detection accuracy and false alarm rates achieved using PCA method

\begin{tabular}{|c|c|c|}
\hline Input images & Using region covariance matrix & Rates \\
\hline \multirow{2}{*}{$\mathbf{1 2 8}$ by $\mathbf{1 2 8}$} & Detection accuracy of target images & $2644 / 2651(99.74 \%)$ \\
\cline { 2 - 3 } & False alarm rates (incorrectly classified clutter images) & $0 / 13445(0 \%)$ \\
\hline \multirow{2}{*}{$\mathbf{6 4}$ by $\mathbf{6 4}$} & Detection accuracy of target images & $2647 / 2651(99.85 \%)$ \\
\cline { 2 - 3 } & False alarm rates (incorrectly classified clutter images) & $0 / 13445(0 \%)$ \\
\hline
\end{tabular}

\section{CONCLUSION}

In this paper, a descriptive feature parameter extraction method from synthetic aperture radar (SAR) images is proposed. The new approach is based on region covariance (RC) and co-difference methods. Experimental results of object detection in MSTAR (moving and stationary target recognition database) are presented. The RC method delivers high detection accuracy and low false alarm rates. It is experimentally observed that the co-difference matrix reaches to better performance than the RC matrix with high target detection and classification performance in SAR images. Furthermore, the experimental results showed that the best detection and classification performance is achieved when the normalized distance measure between co-difference matrices are used to match regions instead of computing eigenvalues. Consequently, the region co-difference method and normalized distance metric approach introduced in this work delivered the highest target detection and classification in SAR images over the other methods including PCA.

\section{ACKNOWLEDGEMENT}

This research is funded in part by TUBITAK.

\section{REFERENCES}

${ }^{[1]}$ Curlander J.C., McConough R.N., Synthetic Aperture Radar, Systems and Signal Processing, Wiley Series in Remote Sensing, New York, 1991.

${ }^{[2]}$ Oliver C., Quegan S., Understanding Synthetic Aperture Radar Images, Scitech Publishing, 2004.

[3] Tuzel O., Porikli F., Meer P., "Region Covariance: A Fast Descriptor for Detection and Classification", European Conference on Computer Vision (ECCV), May 2006.

[4] Tuzel O., Porikli F., Meer P., "Human Detection via Classification on Riemann Manifolds", IEEE Computer Society Conference on Computer Vision \& Pattern Recognition (CVPR), June 2007.

[5] Porikli F., Tuzel O., "Fast Construction of Covariance Matrices for Arbitrary Size Image Windows", IEEE International Conference on Image Processing (ICIP), pp. 1581-1584, October 2006.

${ }^{[6]}$ MSTAR image database: https://sslweb.cis.jhu.edu/ DATASETS/MSTAR/.

[7] Yinan Y., Yuxia Q., Chao L., "Automatic Target Classification - Experiments on the MSTAR SAR Images", SPND/SAWN, 2005.

${ }^{[8]}$ Akgul T., Mingui S., Sclahassi R.J., Cetin A.E., "Characterization of sleep spindles using higher order statistics and spectra", IEEE Transactions on Biomedical Engineering, vol. 47, pp. 997-1009, 2000.

${ }^{[9]}$ Jolliffe I.T., Principal Component Analysis, Series: Springer Series in Statistics, 2nd ed., Springer, NY, 2002.

${ }^{[10]}$ Zhao Q., Principe J., "Support vector machines for SAR automatic target recognition”, IEEE Trans. On AES, Vol. 37, No. 2, pp. 643-654, 2001.

${ }^{[11]}$ Chih-Chung C., Chih-Jen L., A library for SVM - LIBSVM: http://www.csie.ntu.edu.tw/ cjlin/libsvm/, LIBSVM, 2001. 